Check for updates

Cite this: RSC Adv., 2019, 9, 14670

Received 26th January 2019

Accepted 22nd April 2019

DOI: $10.1039 / c 9 r a 00705 a$

rsc.li/rsc-advances

\title{
Metformin promotes proliferation and suppresses apoptosis in Ox-LDL stimulated macrophages by regulating the $\mathrm{miR}-34 \mathrm{a} / \mathrm{Bcl} 2$ axis $\uparrow$
}

\author{
Liuliu Feng, Tianhua Liu, Yuya Yang, Wenying Xiao, Jun Shi, Xiang Mei, Songmei Tian, \\ Xinbing Liu, Hongman Huang and Yanyan Bai (D)*
}

\begin{abstract}
Background: Metformin, an antidiabetic drug, has been reported to be involved in atherosclerosis (AS). In this study, the effects of metformin on oxidized low-density lipoprotein (Ox-LDL)-induced macrophage apoptosis were investigated, and the mechanisms involved in this process were examined. Methods: qRT-qPCR analysis was performed to detect the expression of miR-34a in macrophage cells. Cell proliferation was determined by MTT assays and colony formation assays. Cell apoptosis was assessed by the detection of apoptotic rate and caspase 3 activity. Western blot analysis was performed to evaluate the expression of Bcl2 protein. Results: Metformin treatment promoted proliferation and suppressed apoptosis in macrophages following the treatment of oxidized low-density lipoprotein (Ox-LDL). Metformin could inhibit miR-34a in macrophages. miR-34a overexpression could reverse the effect of metformin on proliferation and apoptosis in Ox-LDL-treated macrophages. Moreover, metformin could increase the expression of the miR-34a target gene Bcl2. Furthermore, metformin treatment exerted the pro-proliferation and anti-apoptosis effect through regulating Bcl2 expression in Ox-LDL-stimulated macrophages. Conclusion: Metformin facilitated proliferation and inhibited apoptosis of macrophages treated with Ox-LDL through the miR-34a/Bcl2 axis, indicating the potential value of metformin in AS therapy.
\end{abstract}

\section{Introduction}

Cardiovascular diseases (CVDs) are a primary cause of death with more than 17.3 million deaths per year worldwide. ${ }^{1}$ Atherosclerosis (AS), a major underlying basis of heart attacks and strokes, accounts for a large proportion of cardiovascular diseases. AS is an inflammatory disease with the characteristics of lipid accumulation, fibrous cap formation and necrotic core generation. $^{2}$ Multiple cell types such as smooth muscle cells (SMCs), endothelial cells (ECs) and macrophages are involved in atherosclerotic lesion formation. ${ }^{3}$ Moreover, previous studies showed that abnormal proliferation, apoptosis and migration of macrophages were closely associated with AS progression., Oxidized low-density lipoprotein (Ox-LDL), a critical risk factor in AS progression, was reported to be able to affect macrophages' apoptosis. ${ }^{6}$ Thus, in the present study, we employed OxLDL-treated macrophages as an AS cell model to investigate the regulatory mechanisms involved in AS.

Metformin is a synthetic dimethyl biguanide and has been a mainstay of therapy for patients with type 2 diabetes mellitus

Department of Cardiology, Shidong Hospital, Yangpu District, Shanghai, 200438, China. E-mail: baiyysdhdc04@126.com; Tel: +86-021-25066666-13101

$\dagger$ Electronic supplementary information (ESI) available. See DOI: $10.1039 / \mathrm{c} 9 \mathrm{ra00705a}$
(T2DM). ${ }^{7}$ Metformin could suppress the expression of proinflammatory factors, and reduce oxidative stress. $^{8-10}$ Several researchers have reported that metformin can slow or reverse the progress of AS. ${ }^{10-13}$ However, the underlying mechanisms of the antiatherogenic role of metformin remain not well elucidated.

MicroRNAs, a class of small non-coding RNA of 22-25 nucleotides, are implicated in the regulation of genes at posttranscriptional level by repressing translation or promoting RNA degradation. ${ }^{14}$ In AS, many microRNAs have been confirmed as vital modulators of pathological processes, such as immune responses, endothelial cell biology, cholesterol and lipid biosynthesis, and lipoprotein metabolism and cholesterol efflux..$^{15}$ Increasing evidence indicated that miR-34a acted as a tumor suppressor in many cancers such as gastric cancer, ${ }^{16}$ breast cancer $^{17}$ and non-small cell lung cancer. ${ }^{18}$ Previous studies revealed that miR-34a was upregulated in atherosclerotic samples and could contribute to AS progression. ${ }^{19-21}$ Nevertheless, how miR-34a was regulated and its functional role in Ox-LDL stimulated macrophages are still unclear. Previous studies reported that metformin could inhibit miR-34a expression in nonalcoholic fatty liver disease and in rat mesangial cells treated with high glucose. ${ }^{22,23}$ However, whether metformin ameliorates the progression of AS through regulating miR34a remains uncertain. 
In our study, we aimed to further explore the underlying roles and molecular mechanisms of metformin in Ox-LDL stimulated macrophages to discover potential therapy targets of AS.

\section{Materials and methods}

\section{Cell culture}

The THP1 macrophage cells were purchased from American Type Culture Collection (ATCC, Manassas, VA, USA) and maintained in RPMI-1640 culture medium supplemented with $10 \%$ FBS (Invitrogen), and $100 \mathrm{U} \mathrm{ml}^{-1}$ penicillin and $100 \mu \mathrm{g} \mathrm{ml}$ streptomycin (Sigma-Aldrich, St. Louis, MO, USA) in a humidified incubator with $5 \% \mathrm{CO}_{2}$ at $37^{\circ} \mathrm{C}$. The cells were treated with $0.5 \mathrm{mM}$ metformin or $50 \mu \mathrm{g} \mathrm{m} \mathrm{m}^{-1} \mathrm{Ox}-\mathrm{LDL}$ for $24 \mathrm{~h}$ for further experiments. Metformin (1,1-dimethylbiguanide, 99.6\% purity) was obtained from Spectrum Chemical manufacturing Corp (New Brunswick, NJ).

\section{Cell transfection and treatment}

Small interference RNA (siRNA) against $\mathrm{Bcl} 2$ (si-Bcl2) and its scramble control (si-con), miR-34a mimic (miR-34a) and its negative control (miR-con), miR-34a inhibitor (anti-miR-34a) and its negative control (anti-miR-con) were designed and synthesized by GenePharma (Guangzhou, China). Cell transfection was performed using lipofectamine 2000 reagent (Invitrogen).

\section{RT-qPCR assays}

Total RNA was extracted using TRIzol® reagent (Invitrogen) and reversely transcribed into cDNA with M-MLV (Invitrogen). Then, the SYBR Green Realtime PCR Master Mix (TOYOBO, Osaka, Japan) were used to detect the expression of miR-34a and Bcl2. U6 snRNA and GAPDH served as endogenous controls for miR$34 \mathrm{a}$ and $\mathrm{Bcl} 2$. The primer sequences were as follows: for miR34a $5^{\prime}$-TGGCAG TGTCTTAGCTGGTTGT-3' (forward) and $5^{\prime}$ GCGAGCACAGAATTAATACGAC-3 ${ }^{\prime}$ (reverse); for $\mathrm{Bcl} 25^{\prime}$ CTGGCATCTTCTCCTTCCAGC-3' (forward) and 5'-ACCTACCCAGCCTCCGTTATC- $3^{\prime}$ (reverse). The relative expression of miR$34 \mathrm{a}$ and $\mathrm{Bcl} 2$ was calculated using the $2^{-\Delta \Delta C_{t}}$ method.

\section{Western blot assays}

Western blot analysis was performed as previously described. ${ }^{24}$ Briefly, the proteins were extracted using RIPA buffer (KeyGEN Biotech, Nanjing, China) and separated by SDS-PAGE gel and transferred to polyvinylidene difluoride (PVDF) membranes (Millipore, Billerica, MA, USA). Then, the membranes were incubated with primary antibodies against $\mathrm{Bcl} 2$ (sc-783, rabbit polyclonal) and $\beta$-actin (sc-1615, goat polyclonal) from Santa Cruz Biotechnology (Santa Cruz, CA, USA). Membranes were washed three times prior to incubation with HRP-conjugated
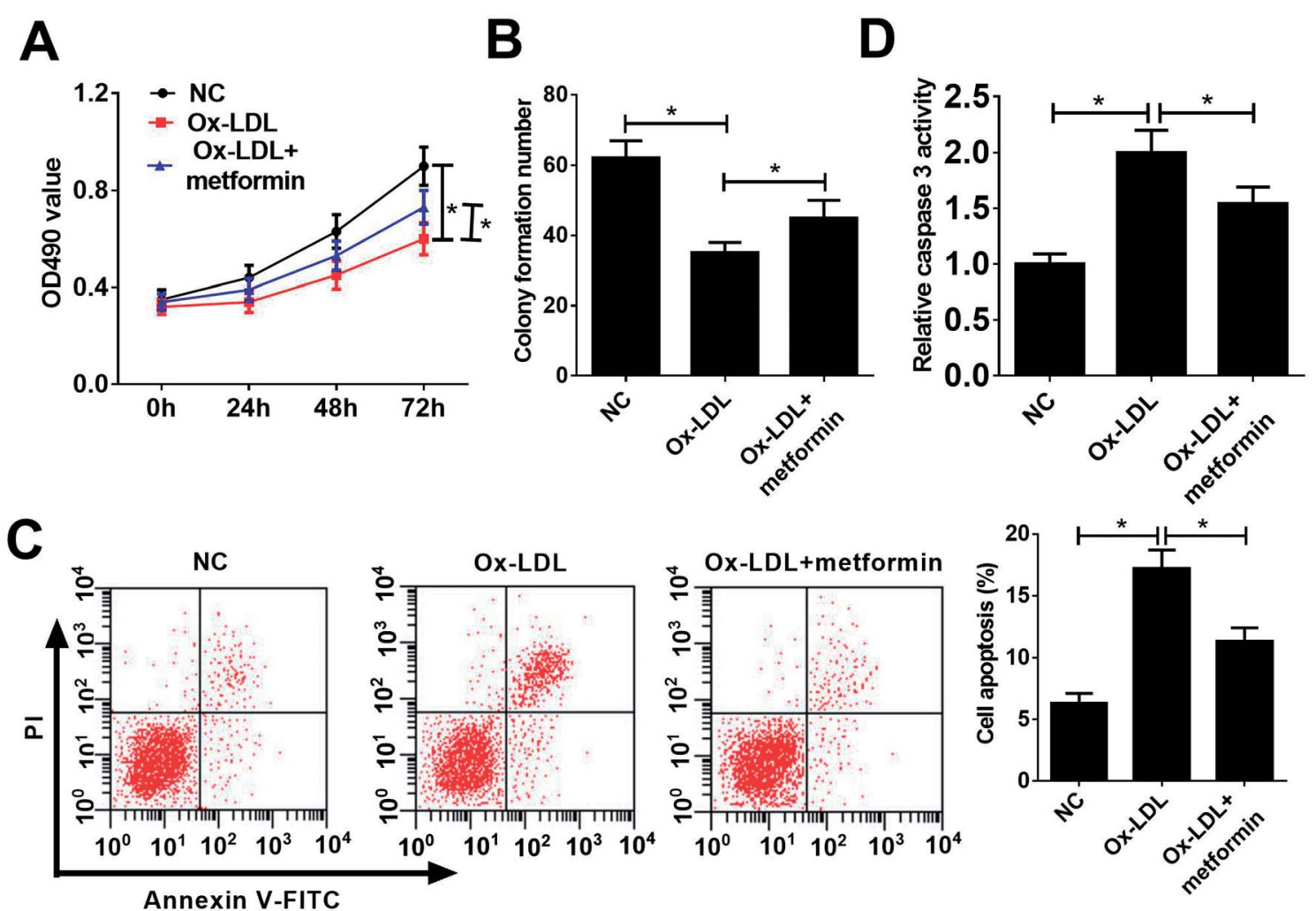

Fig. 1 Metformin promoted proliferation and suppressed apoptosis in Ox-LDL-treated macrophages. Macrophages were treated with $50 \mu \mathrm{g}$ $\mathrm{ml}^{-1} \mathrm{Ox}-\mathrm{LDL}$ or $0.5 \mathrm{mM}$ metformin for $24 \mathrm{~h}$, followed by the detection of proliferation capacity (A), clone formation ability (B), apoptotic rate (C), and caspase 3 activity (D). $* P<0.05$. 
A
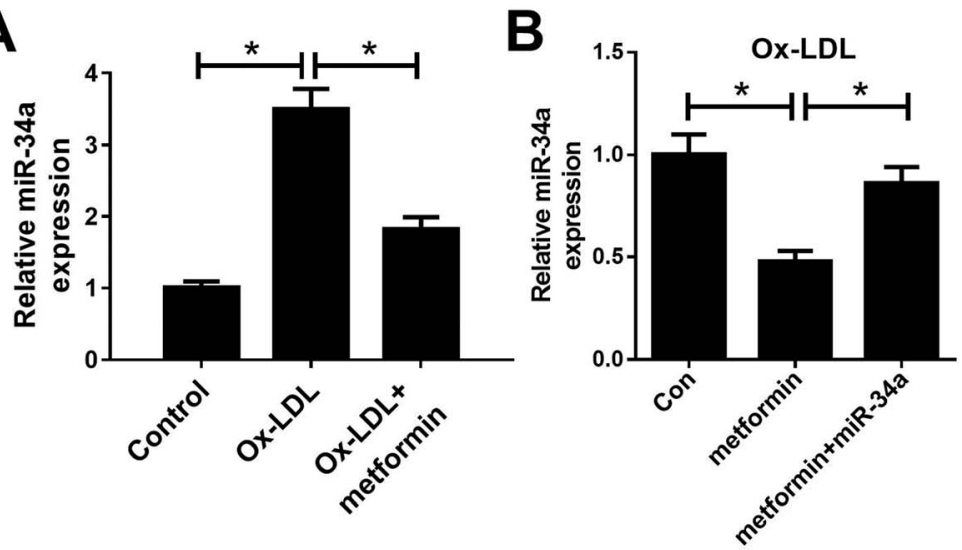

D

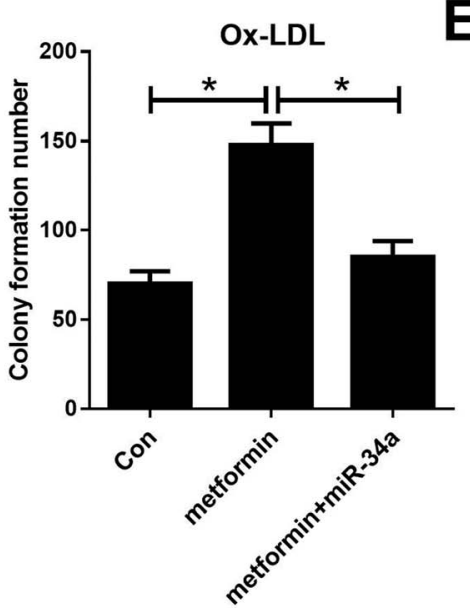

E

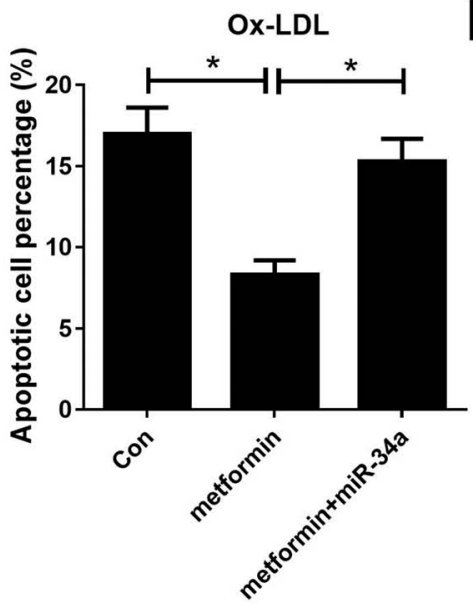

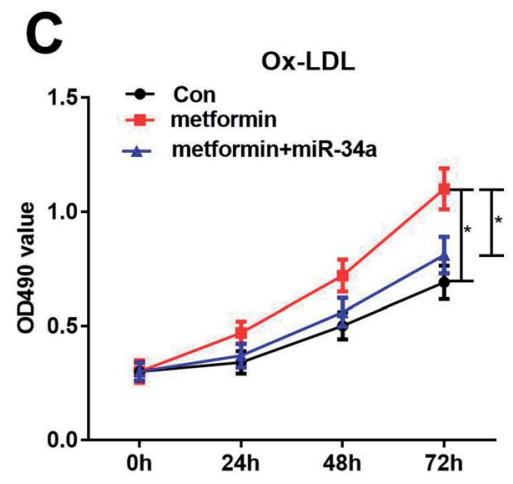

$\mathbf{F}$

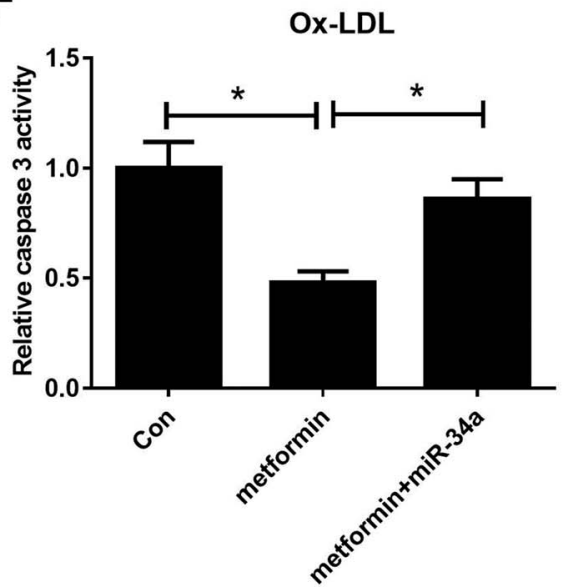

Fig. 2 miR-34a overexpression reversed the effects of metformin on proliferation and apoptosis of Ox-LDL-treated macrophages. (A) qRT-PCR analysis was performed to detect miR-34a expression in Ox-LDL, or Ox-LDL + metformin treated macrophages. Macrophages stimulated with $50 \mu \mathrm{g} \mathrm{ml}^{-1} \mathrm{Ox}-\mathrm{LDL}$ were treated with Con, metformin, or metformin + miR-34a were for $24 \mathrm{~h}$, followed by the measurement of miR-34a expression (B), cell proliferation capability (C), colony formation ability (D), apoptotic rate (E), and caspase 3 activity (F). $* P<0.05$.

anti-mouse secondary antibodies from Santa Cruz Biotechnology. Finally, the protein bands were detected by ECL chemiluminescent regent (Pierce, Rockford, IL, USA).

\section{MTT assays}

Viable macrophages were determined by MTT at $0,24,48$, or $72 \mathrm{~h}$ post-transfection. Briefly, the cells were seeded at density of $2.0 \times 10^{3}$ cells per well into 96-well plates. Following treatment with $50 \mu \mathrm{g} \mathrm{ml}^{-1}$ Ox-LDL and $0.5 \mathrm{mM}$ metformin, $10 \mu \mathrm{l} 3$ (4,5-dimethylthiazol-2-yl)-2,5-diphenyltetrazolium bromide (MTT; Sigma-Aldrich, USA) was added into each well of 96-well plates. The plates were incubated for $4 \mathrm{~h}$ at $37{ }^{\circ} \mathrm{C}$. Then the absorbance at $490 \mathrm{~nm}$ was determined to evaluate cell proliferation.

\section{Colony formation assays}

Transfected macrophages $\left(2.0 \times 10^{4}\right.$ cells per well $)$ were seeded in a 12-well plate and cultured for 15 days in complete medium. Then cells were fixed with methanol and stained with $0.1 \%$ crystal violet solution (Sigma-Aldrich). At last, the total number of colonies with a minimum of 50 cells was detected using a microscope.

\section{Flow cytometric analysis}

The apoptosis rate of macrophages was evaluated using Annexin V-FITC/PI Apoptosis Detection Kit (KeyGEN Biotech, Nanjing, China) as described previously. ${ }^{25}$ Briefly, macrophages were stained with $5 \mu \mathrm{l}$ Annexin V-FITC $\left(1 \mu \mathrm{g} \mathrm{ml}{ }^{-1}\right)$ for $10 \mathrm{~min}$ and $5 \mu \mathrm{l}$ propidium iodide (PI) $\left(1 \mu \mathrm{g} \mathrm{ml}^{-1}\right)$ for $5 \mathrm{~min}$ in the dark at room temperature. The apoptotic rates were analyzed using CellQuest software (BD Biosciences, Franklin Lakes, NJ, USA).

\section{Caspase 3 activity detection}

Macrophages were treated with $50 \mu \mathrm{g} \mathrm{ml}^{-1}$ Ox-LDL and $0.5 \mathrm{mM}$ metformin for $24 \mathrm{~h}$. Cells were lysed, and the protein concentration was measured using the Bradford Protein Assay Kit (Beyotime Biotechnology, Shanghai, China). Then, equal amount of proteins was incubated with reaction buffer containing enzyme-specific substrates for caspase 3 at $37^{\circ} \mathrm{C}$ for $1 \mathrm{~h}$. The absorbance of $405 \mathrm{~nm}$ was detected using a microplate reader (Molecular Devices, Sunnyvale, California, USA) using 
A
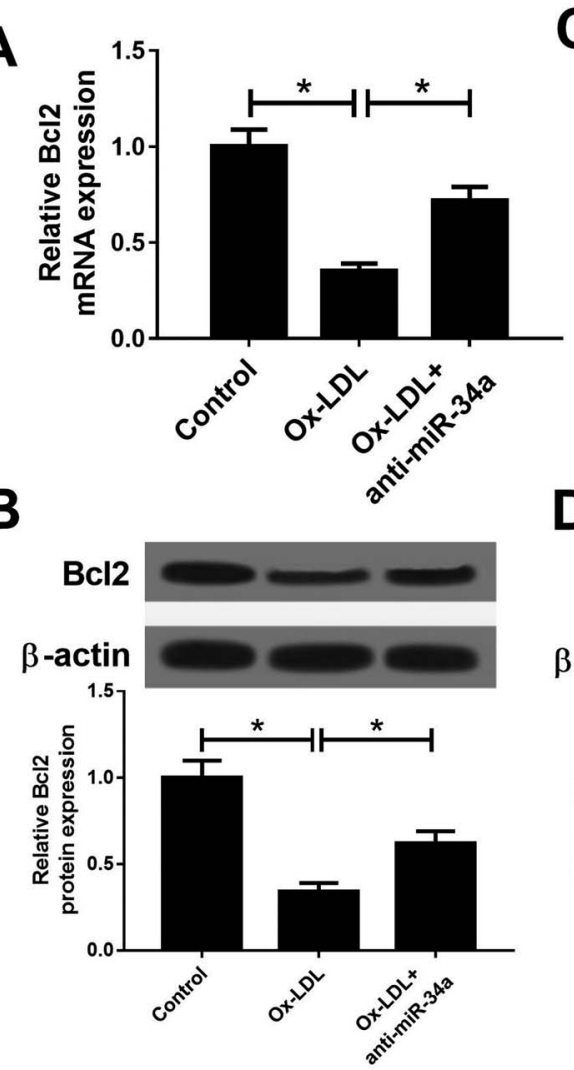

C

D

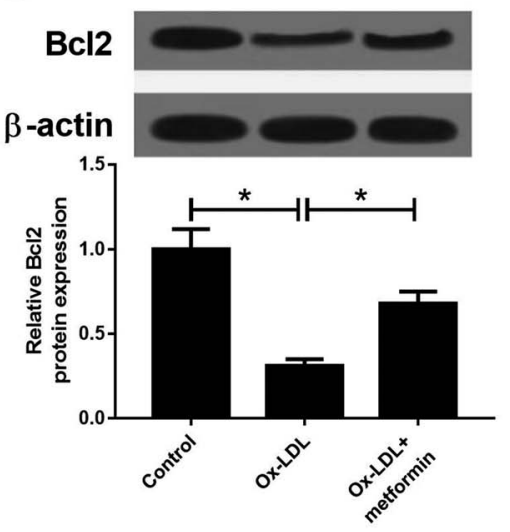

E

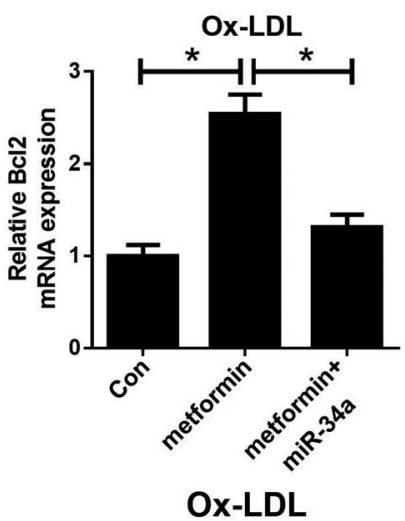

\section{$\mathrm{Bcl} 2$}

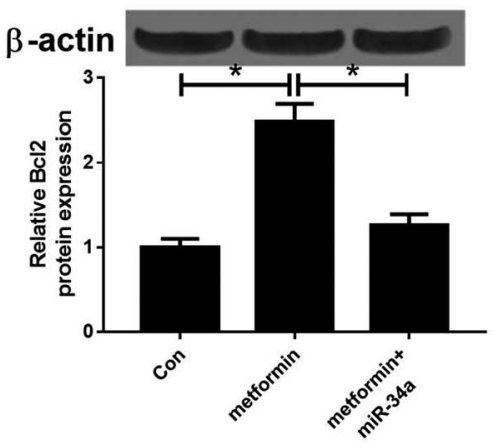

Fig. 3 Metformin promoted the expression of miR-34a target Bcl2. (A and B) The Bcl2 mRNA and protein expression was determined in macrophages treated with Ox-LDL or Ox-LDL + anti-miR-34a. (C and D) The Bcl2 mRNA and protein expression was determined in macrophages treated with $\mathrm{Ox}-\mathrm{LDL}$ or $\mathrm{Ox}-\mathrm{LDL}+$ metformin. (E and F) The Bcl2 mRNA and protein expression was determined in Ox-LDL treated macrophages treated with Con, metformin, or metformin + miR-34a. ${ }^{*} P<0.05$.

the Caspase 3 Assay Kit (Calbiochem, San Diego, California, USA).

\section{Statistical analysis}

All data were presented as mean \pm standard deviation (SD). The comparisons of data in different groups were performed using Student's $t$-test or one-way variance analysis. $P<0.05$ was statistically significant.

\section{Results}

Metformin promoted proliferation and suppressed apoptosis in Ox-LDL-treated macrophages

To investigate the functional role of metformin in AS process, we explored the effect of metformin on proliferation and apoptosis in Ox-LDL-treated macrophages. Macrophages were treated with $50 \mu \mathrm{g} \mathrm{m} \mathrm{m}^{-1} \mathrm{Ox}-\mathrm{LDL}$ and with or without $0.5 \mathrm{mM}$ metformin for $24 \mathrm{~h}$, followed by measurement of cell proliferation ability and apoptotic rate. MTT assays revealed that OxLDL could suppress macrophages proliferation, however, metformin treatment weakened the inhibitory effect of Ox-LDL on macrophages proliferation (Fig. 1A). Moreover, colony formation assays also demonstrated that metformin significantly improved colony formation potential of Ox-LDL-treated macrophages (Fig. 1B). Additionally, flow cytometry assays showed that Ox-LDL could induced macrophages apoptosis, nevertheless, metformin markedly lowered the apoptotic rate of Ox-LDL-treated macrophages (Fig. 1C). Consistently, metformin treatment resulted in a remarkable reduction of caspase 3 activity in Ox-LDL-treated macrophages (Fig. 1D). In summary, these results suggested that metformin facilitated proliferation and suppressed apoptosis in Ox-LDL-treated macrophages.

miR-34a overexpression reversed the effects of metformin on proliferation and apoptosis of Ox-LDL-treated macrophages

Previous studies reported that metformin could down-regulate miR-34a expression in high glucose condition and downregulation of miR-34a could suppress Ox-LDL-induced apoptosis. $^{26,27}$ Hence, we presumed that metformin may promote proliferation and suppressed apoptosis in Ox-LDL stimulated macrophages through regulating miR-34a expression. To confirm whether the pro-proliferation and antiapoptosis effect of metformin was mediated by miR-34a, we first detected miR-34a expression in macrophages with Ox-LDL and metformin treatment. As expected, Ox-LDL could increase miR-34a expression, which was weakened by metformin treatment (Fig. 2A). Then, we treated Ox-LDL stimulated macrophages with metformin or metformin + miR-34a. qRT-PCR 

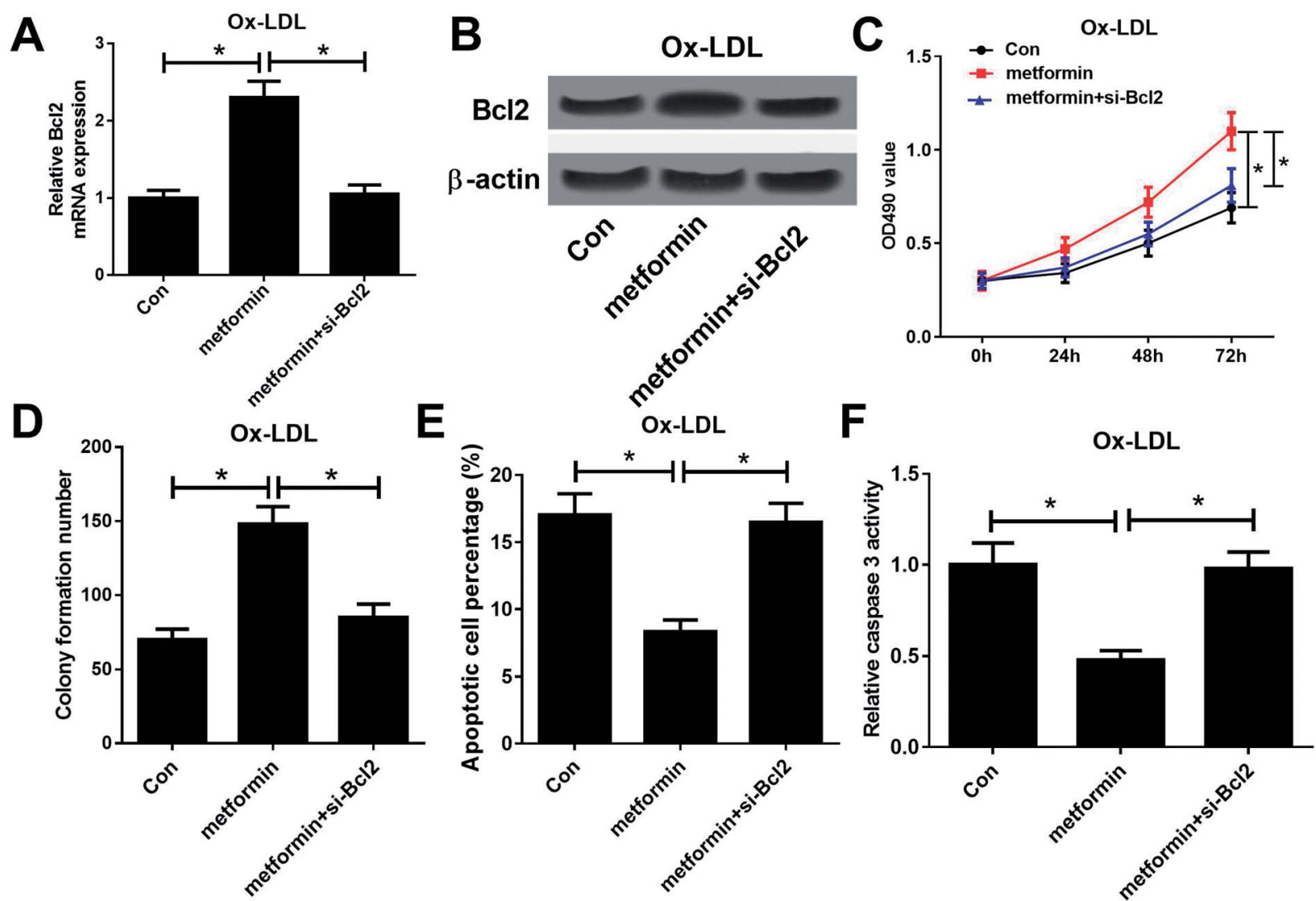

Fig. 4 Metformin promoted proliferation and inhibited apoptosis by regulating miR-34a/Bcl2 axis in Ox-LDL-treated macrophages. Macrophages were treated with $50 \mu \mathrm{g} \mathrm{ml}^{-1} \mathrm{Ox}-\mathrm{LDL}$ and Con, metformin, or metformin $+\mathrm{si}-\mathrm{Bcl} 2$ for $24 \mathrm{~h}$, followed by the determination of Bcl2 mRNA and protein levels (A and B), cell proliferation ability (C), colony formation capability (D), apoptotic rate (E), and caspase 3 activity (F). $* P<0.05$.

analysis indicated that introduction of miR-34a mimics could abolish the inhibitory effect of metformin on miR-34a expression (Fig. 2B). MTT and colony formation assays further showed that the enhancive effect of metformin on cell growth was greatly abrogated by miR-34a overexpression (Fig. 2C and D). As expected, miR-34a overexpression markedly abated metforminmediated apoptosis inhibition (Fig. 2E and F). Taken together, these results indicated that metformin exerted its proproliferation and anti-apoptosis effect in Ox-LDL-treated macrophages through inhibiting miR-34a expression.

\section{Metformin elevated the expression of miR-34a target Bcl2}

It is well known that miRNAs exert their functions through targeting special mRNAs. Bcl2, a known target of miR-34a, has been reported to be implicated with AS progression. ${ }^{28}$ Hence, we further explored whether metformin positively regulated $\mathrm{Bcl} 2$ through inhibiting miR-34a expression. As displayed in Fig. 3A and B, miR-34a inhibition could elevate the reduced Bcl2 mRNA and protein expression caused by Ox-LDL in macrophages. Consistently, metformin increased Bcl2 mRNA and protein levels in Ox-LDL treated macrophages (Fig. $3 \mathrm{C}$ and D). Additionally, miR-34a overexpression relieved metformin-mediated increase on Bcl 2 mRNA and protein levels in Ox-LDL treated macrophages (Fig. 3E and F). Collectively, these data indicated that metformin could elevate Bcl2 expression through suppressing miR-34a in Ox-LDL treated macrophages.

\section{Metformin promoted by regulating miR-34a/Bcl2 axis in Ox-} LDL-treated macrophages

To further examine whether the effect of metformin on proliferation and apoptosis was mediated by miR-34a/Bcl2 axis in OxLDL-stimulated macrophages, Ox-LDL treated macrophages were treated with Con, metformin or metformin + si-Bcl2. Western bolt analysis revealed that introduction of si-Bcl2 weakened the increased Bcl2 mRNA and protein levels caused by metformin treatment in macrophages (Fig. 4A and B). Moreover, metformin-mediated enhancement on cell growth was greatly declined by Bcl2 knockdown in macrophages (Fig. 4C and D). Furthermore, Bcl2 silencing markedly abated metformin-mediated apoptosis inhibition macrophages (Fig. 4E and F). These data demonstrated that metformin exerted its pro-proliferation and anti-apoptosis effect by regulating miR-34a/Bcl2 axis in Ox-LDL-treated macrophages.

\section{Discussion}

Although much progress has been made in the pathogenesis and therapeutic options of AS, AS is still a major threaten of human health with a high morbidity and mortality worldwide. ${ }^{29}$ 
Previous studies have demonstrated that metformin, an antidiabetic drug, has obvious anti-atherogenic properties, ${ }^{\mathbf{1 2 , 3 0 - 3 2}}$ but how metformin exerts these effects is unclear. In the present study, we investigated the effects of metformin treatment on the progression of atherogenesis using an Ox-LDL-induced atherosclerotic cell model. Our results suggested that metformin significantly attenuated the progression of atherosclerosis by promoting proliferation and suppressing apoptosis in Ox-LDL stimulated macrophages through miR-34a/Bcl2 axis.

In recent years, many studies have revealed that metformin has many excess effects on blood vessels besides its hypoglycemic effect. ${ }^{33,34}$ Metformin was identified to be the only hypoglycemic drug lowering the risk of cardio-cerebrovascular complications of T2DM patients. ${ }^{35}$ Increasing evidence has suggested that metformin is implicated with the progression of AS. For example, metformin could suppress diabetes-related atherosclerosis via inhibiting Drp1-mediated mitochondrial fission. ${ }^{13}$ Furthermore, metformin could ameliorate the proinflammatory state in patients with carotid artery atherosclerosis. ${ }^{36}$ Metformin could also reduce lipid accumulation in macrophages by inhibiting FOXO1-mediated transcription of fatty acid-binding protein $4 .{ }^{37}$ Another study demonstrated that metformin inhibits macrophage cholesterol biosynthesis rate through attenuated oxidative stress. ${ }^{38}$ Since macrophages play a vital role in the progress of atherosclerosis, we assume that metformin attenuated atherosclerosis may be related to the inhibition of apoptosis in macrophages. Our findings revealed that metformin treatment promoted proliferation and suppressed apoptosis in Ox-LDL stimulated macrophages, which suggested that metformin could attenuate AS progression.

In this study, we further investigate the mechanism underlying the inhibition of AS progression by metformin. Previous studies reported metformin could regulate the expression of miRNAs, which exerts vital roles in various diseases. Therefore, we presumed that metformin may attenuate AS through modulating AS-related miRNAs. A previous study reported that metformin could regulate miR-34a expression in rat mesangial cells cultured with high glucose ${ }^{26}$ Moreover, downregulation of miR-34a suppressed Ox-LDL-induced apoptosis and oxidative stress in human umbilical vein endothelial cells. ${ }^{27}$ However, the role of miR-34a in Ox-LDL stimulated macrophages is still unclear. Hence, we further explored whether metformin promoted proliferation and suppressed apoptosis in Ox-LDL stimulated macrophages through regulating miR-34a expression. Our study found that metformin treatment decreased miR-34a expression in macrophages. Furthermore, miR-34a overexpression could reverse the inductive effect of metformin on proliferation and the inhibitory effect of metformin on apoptosis in Ox-LDL stimulated macrophages. Similarly, a previous study revealed that downregulation of miR-34a promoted endothelial cell growth and suppresses apoptosis in Ox-LDL-treated human aortic endothelial cells. ${ }^{27}$ All these data demonstrated that metformin ameliorated AS through inhibiting miR-34a expression.

Emerging evidence shows that miRNAs exert functions by regulating translation or stability of target mRNAs. Hence, the potential target mRNAs of miR-34a was further investigated.
$\mathrm{Bcl} 2$, an anti-apoptotic gene, was reported to be a target of miR$34 \mathrm{a},{ }^{27}$ which has been reported to be involved in AS progress. ${ }^{39,40}$ In this work, we found that metformin could elevate $\mathrm{Bcl} 2$ expression through inhibiting miR-34a expression. Furthermore, Bcl 2 knockdown could abolished the inhibitory effect of metformin treatment on AS. In a word, metformin ameliorated AS through modulating miR-34a/Bcl2 axis.

In summary, our study demonstrated that metformin promoted proliferation and suppressed apoptosis in ox-LDLtreated macrophages by elevating $\mathrm{Bcl} 2$ expression via inhibiting miR-34a. This finding elucidated the involvement of metformin/miR-34a/Bcl2 regulatory axis in AS progression, which provided a promising target for AS treatment.

\section{Conflicts of interest}

The authors declare that they have no competing interests.

\section{Acknowledgements}

The research was approved by the Project of Shanghai Municipal Commission of Health and Family Planning.

\section{References}

1 L. J. Laslett, P. Alagona, B. A. Clark, J. P. Drozda, F. Saldivar, S. R. Wilson, C. Poe and M. Hart, J. Am. Coll. Cardiol., 2012, 60, S1-S49.

2 S. Carr, A. Farb, W. H. Pearce, R. Virmani and J. S. T. Yao, J. Vasc. Surg., 1996, 23, 755.

3 A. C. Doran, M. Nahum and C. A. Mcnamara, Arterioscl. Throm. Vas., 2008, 28, 812.

4 B. Wang, Z. Ge, Z. Cheng and Z. Zhao, Biol. Open, 2017, 6, 489-495.

5 X. Zhuang, R. Li, A. Maimaitijiang, R. Liu, F. Yan, H. Hu, X. Gao and H. Shi, J. Cell. Biochem., 2018, 120, 6304-6314.

6 Y. Chen, M. Li, Y. Zhang, M. Di, W. Chen, X. Liu, F. Yu, H. Wang, X. Zhen and M. Zhang, Biochem. Biophys. Res. Commun., 2018, 501, 336-342.

7 C. J. Bailey and R. C. Turner, N. Engl. J. Med., 1996, 334, 574579.

8 W. Xu, Y. Y. Deng, L. Yang, S. Zhao, J. Liu, Z. Zhao, L. Wang, P. Maharjan, S. Gao and Y. Tian, Transl. Res., 2015, 166, 451458.

9 D. Pavlović, R. Kocić, G. Kocić, T. Jevtović, S. Radenković, D. Mikić, M. Stojanović and P. B. Djordjević, Diabetes, Obes. Metab., 2010, 2, 251-256.

10 J. C. Mamputu, N. F. Wiernsperger and G. Renier, Diabetes Metab., 2003, 29, 6S71-6S76.

11 F. Kathleen, A. Suhny, L. Hang, S. Eleni, S. Rachel, M. Theresa, H. Linda, T. Martin and G. Steven, AIDS, 2012, 26, 587-597.

12 L. Fei, G. Yuan, G. Ruan, J. Long, X. Zheng, X. Qin, S. Zhao, D. Peng, Z. Fang and X. Li, Sci. Rep., 2017, 7, 2169.

13 Q. Wang, M. Zhang, G. Torres, S. Wu, C. Ouyang, Z. Xie and M. H. Zou, Diabetes, 2017, 66, 193-205. 
14 M. Georges and C. Charlier, Curr. Opin. Genet. Dev., 2007, 17, 166-176.

15 H. Giral, A. Kratzer and U. Landmesser, Best Pract. Res., Clin. Endocrinol. Metab., 2016, 30, 665-676.

16 Y. Peng, J. J. Guo, Y. M. Liu and X. L. Wu, Biosci. Rep., 2014, DOI: 10.1042/BSR20140020.

17 M. Xu, D. Li, C. Yang and J. S. Ji, Cell. Physiol. Biochem., 2018, 46, 1286-1304.

18 J. Y. Zhou, X. Chen, J. Zhao, Z. Bao, X. Chen, P. Zhang, Z. F. Liu and J. Y. Zhou, Cancer Lett., 2014, 351, 265-271.

19 E. Raitoharju, L.-P. Lyytikäinen, M. Levula, N. Oksala, A. Mennander, M. Tarkka, N. Klopp, T. Illig, M. Kähönen and P. J. Karhunen, Atherosclerosis, 2011, 219, 211-217.

20 X. Zhong, P. Li, J. Li, R. He, G. Cheng and Y. Li, Int. J. Mol. Med., 2018, 42, 1134-1144.

21 G. Su, G. Sun, H. Liu, L. Shu and Z. Liang, Heart Vessels, 2018, 33, 1-10.

22 K. Akiko, M. Asahiro, I. Hisakazu, T. Joji, S. Teppei, T. Miwa, T. Yuka, F. Koji, K. Kiyohito and M. Emiko, Int. J. Mol. Med., 2015, 35, 877-884.

23 C. Wu, N. Qin, H. Ren, M. Yang, S. Liu and Q. Wang, Int. J. Endocrinol., 2018, 6462793, DOI: 10.1155/2018/6462793.

24 K. F. Lei, Y. F. Wang, X. Q. Zhu, P. C. Lu, B. S. Sun, H. L. Jia, N. Ren, Q. H. Ye, H. C. Sun and L. Wang, BMC Cancer, 2007, 7, 172.

25 W. J. Wang, Y. Yao, L. L. Jiang, T. H. Hu, J. Q. Ma, Z. J. Liao, J. T. Yao, D. F. Li, S. H. Wang and K. J. Nan, PLoS One, 2013, 8, e76596.

26 C. Wu, N. Qin, H. Ren, M. Yang, S. Liu and Q. Wang, Int. J. Endocrinol., 2018, 2018, 1-15.
27 X. Zhong, P. Li, J. Li, R. He, G. Cheng and Y. Li, Int. J. Mol. Med., 2018, 42, 1134-1144.

28 E. Thorp, Y. Li, L. Bao, P. M. Yao, G. Kuriakose, J. Rong, E. A. Fisher and I. Tabas, Arterioscler., Thromb., Vasc. Biol., 2009, 29, 169-172.

29 W. Christian and N. Heidi, Nat. Med., 2011, 17, 1410-1422.

30 F. Farshad, S. Gloria, P. Nikolay, X. Shiqin, H. Lula, F. Baowei and A. R. Wayne, J. Am. Heart Assoc., 2014, 3, A2040.

31 K. Adriaan, D. J. Jolien, L. Philippe, B. Daniel, M. G. Wulffelé, A. J. M. Donker and C. D. A. Stehouwer, Arch. Intern. Med., 2009, 169, 616.

32 P. M. Ridker, Lancet, 2014, 384, 607-617.

33 A. R. Cameron, V. L. Morrison, D. Levin, M. Mohan, C. Forteath, C. Beall, A. D. Mcneilly, D. J. K. Balfour, T. Savinko and A. K. F. Wong, Circ. Res., 2016, 119, 652-665. 34 S. Gillessen, C. Gilson, N. James, A. Adler, M. R. Sydes and N. Clarke, Eur. Urol., 2016, 70, 906-908.

35 A. S. G. W. Committee, Diabetes Care, 2016, 39, 701.

36 W. Xu, Y. Y. Deng, L. Yang, S. Zhao, J. Liu, Z. Zhao, L. Wang, P. Maharjan, S. Gao and Y. Tian, Transl. Res., 2015, 166, 451458.

37 J. Song, P. Ren, L. Zhang, X. L. Wang, L. Chen and Y. H. Shen, Biochem. Biophys. Res. Commun., 2010, 393, 89-94.

38 M. Korengluzer, M. Aviram and T. Hayek, Biochem. Biophys. Res. Commun., 2013, 439, 396-400.

39 Y. Yu, Q. Liu, S. Guo, Q. Zhang, J. Tang, G. Liu, D. Kong, J. Li, S. Yan, R. Wang, P. Wang, X. Su and Y. Yu, J. Cell. Mol. Med., 2017, 21, 3540-3551.

40 R. Wu, S. Tang, M. Wang, X. Xu, C. Yao and S. Wang, PLoS One, 2016, 11, e0167052. 